\title{
Historia de los Estudios sobre Medios,
}

\section{en plural}

David W. Park

Lake Forest College, park@lfc.edu

Jefferson Pooley

Muhlenberg College, pooley@muhlenberg.edu

\section{Peter Simonson}

University of Colorado Boulder, peter.simonson@colorado.edu

\section{Traducción de Raúl Fuentes-Navarro}

Lanzamos la publicación de Historia de los Estudios sobre Medios con una concesión que es al mismo tiempo una confesión: el objeto de la revista, la historia de los estudios sobre medios, es poroso y extensible, casi amorfo. La razón principal es que los "estudios sobre medios" no tienen un referente compartido. La expresión se impuso solamente - como un sustantivo singular que designa a un campoa finales de los años sesenta y en los setenta, mucho después de que el estudio de los medios y la comunicación se enraizara de diferentes maneras en la universidad. Había "estudios sobre medios", en plural, pero no existía algo como un campo con ese nombre. Incluso hoy en día muchos académicos que trabajan en temas relacionados con los medios y la comunicación, si se les pidiera que nombraran su campo, darían alguna otra respuesta. Por su parte, los académicos que se autodefinen como practicantes de "estudios sobre medios", podrían entrecerrar los ojos ante la definición de su campo por parte de sus presuntos colegas.

History of Media Studies, vol. 1, 2021
David W. Park, Jefferson Pooley, and Pete Simonson, "Historia de los Estudios sobre Medios, en plural," History of Media Studies 1 (2021), https://doi.org/ 10.32376/d895aoea.7649e543.

(c) (i) () 
Consideramos que los deslices del término son una ventaja editorial. La premisa de la revista es que lo que cuenta como historia de los estudios sobre medios está de por sí en juego. La tarea de nuestros autores, dicho de otro modo, es definir el alcance del campo mediante la acumulación de argumentos ejemplares en sus artículos. Hay etiquetas disciplinares con referentes más estables - como "investigación en comunicación" o "estudios de cine" - , pero cada una de ellas es una delimitación parcial. Por eso una de las virtudes de los "estudios sobre medios" es su porosidad y ambivalencia.

Otra virtud proviene del propio concepto de medios (de comunicación). Con raíces que se remontan al menos hasta la Ilustración, "medios" y "mediación" se han vuelto cada vez más fundamentales para entender las texturas sociales del mundo moderno. ${ }^{1}$ Son conceptos amplios, centrales a todos los procesos comunicativos, vinculados de una manera a su materialidad, y de otra, a los procesos de representación, articulación, traducción e intermediación. La forma dominante, "medios" en plural —como órganos comerciales de comunicación de masas-, se afianzó apenas en la década de 1950, en el apogeo de la era de la radiodifusión. ${ }^{2}$ Invocamos a "los medios", en su rico sentido católico, como una invitación a ventilar lo que podría ser la historia de su estudio.

Como punto de partida, esperamos publicar trabajos que abarquen la historia de las humanidades y de las ciencias sociales. Esperamos que los estudios académicos sobre la historia de la investigación en comunicación, los estudios culturales, los estudios cinematográficos, la ciencia de la información, los estudios de periodismo, el discurso (speech) y la retórica, figuren en la mezcla editorial. Muchos de los temas, discursos e instituciones que nuestros autores investigan no encajarán tan fácilmente. Los estudiosos de los medios se han identificado con docenas de otras disciplinas, a lo largo de contextos locales y nacionales apenas representados en la literatura publicada. Además, muchos estudiantes de medios nunca han estado afiliados a universidades sino que trabajan para empresas comerciales, agencias gubernamentales o grupos sin fines de lucro, o están integrados a movimientos sociales. Nuestro propósito es publicar también sus historias.

\section{Exclusiones en la historiografía de los estudios sobre medios}

La misión de la revista está determinada por el momento histórico. Nuestros campos se están enfrentando tardíamente a las herencias y consecuencias actuales de una serie de inequidades estructurales que se entrecruzan: su blanquitud y el patriarcado; las hegemonías de la lengua inglesa y de las formas estadounidenses de pensamiento e
${ }^{1}$ John Guillory, "Enlightening Mediation", en This is Enlightenment, ed. por Clifford Siskin y William Warner (Chicago: University of Chicago Press, 2010).

${ }^{2}$ Anna Shechtman, "Command of Media's Metaphors", Critical Inquiry 47, no. 4 (2021). 
investigación; los efectos persistentes del colonialismo y la antinegritud; las muchas formas de jerarquización y exclusión que emanan de los centros y las periferias de todo el mundo, y la colonización neoliberal de universidades y publicaciones académicas, por nombrar algunas de las más destacadas. Hasta ahora, la mayor parte de los textos críticos sobre estos temas se ha centrado, con razón, en sus manifestaciones contemporáneas. Historia de los Estudios sobre Medios busca constituirse en un foro para investigar, además, la dinámica histórica por la que los campos de estudio de los medios han llegado a este punto, tanto en términos de los patrones sociales dominantes que los han definido como de la mezcla de conciencia crítica y prácticas alternativas que podrían dar lugar a otras maneras de avanzar en nuestro trabajo intelectual. Más allá de publicar estas genealogías del presente, la revista también se compromete con sus propias prácticas alternativas y con el apoyo a los esfuerzos colectivos que han dinamizado el momento actual.

Historia de los Estudios sobre Medios tiene como objetivo contribuir a lo que podría llamarse el descentramiento de los centros en estos patrones interrelacionados de marginación. Esto no será un trabajo fácil ni sencillo. Los hábitos heredados del campo están profundamente arraigados en sus prácticas - y en los cuerpos y mentes de quienes trabajamos en él. Estos hábitos atraviesan la geopolítica, el lenguaje, la ideología, la economía política y las interseccionalidades de la identidad social. Todo esto continúa desarrollándose en un momento particularmente peligroso de la historia, cuando las desigualdades profundas, las crisis políticas y el cambio climático amenazan la posibilidad de un futuro compartido y habitable en el planeta. No nos hacemos muchas ilusiones sobre la importancia de una nueva revista académica en estos contextos, y no queremos exagerar la importancia de los esfuerzos que realiza. Dicho esto, creemos que las revistas refractan las prácticas y las distorsiones más amplias de los campos académicos en un microcosmos, y que deberían hacer su parte para demandar más del presente. Una revista nueva puede al menos abrir un espacio modesto para pensar y hacer las cosas de manera diferente, sirviendo como un pequeño laboratorio que podría dar forma a esfuerzos en otros lugares.

Uno podría preguntarse cómo una revista cuyos editores fundadores son tres cishombres blancos de Estados Unidos puede esperar de buena fe contribuir a esos esfuerzos. Nos tomamos esas dudas en serio y, de hecho, las compartimos. La respuesta breve es que no podemos esperar hacer mucho por nuestra cuenta. Estamos de acuerdo con el argumento de Mohan Dutta de que debemos diseñar y poner en práctica diferentes tipos de comunicación si queremos transformar las formas heredadas de producir conocimientos 
académicos y constituir nuestros campos. ${ }^{3}$ En el proyecto de Historia de los Estudios sobre Medios, esto comienza con nuestro Consejo Editorial, que será un órgano más participativo que muchas entidades de este tipo, dando forma activa al trabajo que hacemos y a la manera en que lo hacemos. Somos muy afortunados de contar con un consejo geográficamente diverso y de gran talento, cuyo número de miembros seguirá creciendo. Los ensayos de lanzamiento que han escrito aquí son los signos más visibles de su participación en esta iniciativa, pero de ninguna manera los únicos. Los miembros del consejo nos ayudan a imaginar y a poner en marcha una revista guiada por los ideales de cuidado, oficio y amistad colegiada más allá de las fronteras, dirigida hacia la investigación de la historia de los estudios sobre medios en toda su complejidad alrededor del mundo. En este contexto, los editores de Historia de los Estudios sobre Medios vemos nuestro papel como facilitadores de un proceso colectivo de traer algo nuevo al mundo. Estamos comprometidos a aprovechar los privilegios estructurales que podamos tener para contribuir a esa labor y avanzar a través de la apertura dialógica a la diferencia y la crítica.

El antropólogo de origen colombiano Arturo Escobar, inspirado por los zapatistas mexicanos, ha propuesto diseñar un mundo para "el pluriverso, un mundo en el que quepan muchos mundos". ${ }^{4}$ ¿Qué supondrían las historias de los estudios sobre medios escritas para un pluriverso y cómo podría diseñarse la revista para facilitarlas? No pretendemos saberlo, pero queremos crear espacios para la experimentación y tenemos algunos compromisos de entrada. Uno de ellos tiene que ver con el lenguaje, que como señala Susana Martínez Guillem es "un eje fundamental de las relaciones de poder" que debería figurar en los esfuerzos por remodelar el campo contemporáneo. ${ }^{5}$ La marginación de los académicos y de los estudios que trabajan en lenguas distintas del inglés es obvia para cualquiera que se encuentre fuera del centro de exclusividad de este idioma en los campos académicos dominados por Estados Unidos. Como un paso modesto en otra dirección, Historia de los Estudios sobre Medios revisará y publicará manuscritos tanto en español como en inglés, y esperamos ampliarnos en el futuro más allá de estos dos idiomas.

Un segundo compromiso es apoyar la escritura de historias de los estudios sobre medios de todo el mundo, en especial del Sur Global y de otras regiones que han sido periféricas, cuando mucho, en la historiografía en lengua inglesa hasta hoy. Esto significa más que simplemente generar un registro más completo de la historia de los estudios sobre medios en todo el mundo, aunque creemos que es un objetivo importante. Significa también apoyar las historiografías análogas a la "teoría desde el Sur", que podrían reorientar las formas heredadas de escribir las historias de los estudios sobre medios, o al
${ }^{3}$ Mohan J. Dutta, "Whiteness, Internationalization, and Erasure: Decolonizing Futures from the Global South", Communication and Critical/Cultural Studies 17, no. 2 (2020).

\footnotetext{
${ }^{5}$ Susana Martínez Guillem, "Sacando la Lengua in Rhetorical Theory and Criticism", Rhetoric, Politics \& Culture 1, no. 1 (2021), 45. Véase también Silvio Waisbord, "Communication Studies without Frontiers? Translation and Cosmopolitanism across Academic Cultures", International Journal of Communication 10 (2016), y Ana Cristina Suzina, "English as Lingua Franca: On the Sterilisation of Scientific Work", Media, Culture $\mathcal{E}$ Society 43 , no. 1 (2021).
} 
menos ayudar a crear algunos "espacios comunes con margen para la diferenciación", propuestos por los defensores del cosmopolitismo académico. ${ }^{6}$ Esto debe hacerse de manera que no se perpetúe la práctica de que los académicos del Norte Global hablen de los contextos del Sur sin incluir las voces de esas regiones. ${ }^{7}$ Asimismo, el compromiso implica "provincializar" las particularidades tradicionalmente no marcadas que se han enmascarado como universales, o al menos como las historias de base dadas por sentadas que, de manera intencional o no, se han presentado como historias del campo, cuando en realidad son las historias de un puñado de académicos exitosos, institucionalmente bien ubicados y abrumadoramente de género masculino que publicaron en inglés o fueron lo suficientemente prominentes como para que su trabajo fuera traducido.

Esto, a su vez, da pie a un tercer compromiso de la revista: centrarse no solo en los estudios sobre medios tal y como se han desarrollado al interior de las instituciones académicas, ya sean centrales o periféricas, sino también en lo que llamamos "tradiciones alternas" de la investigación reflexiva sobre los medios, de nuevo concebida en sentido amplio. ¿Qué conceptualizaciones, prácticas educativas, marcos normativos y formas de práctica social reflexiva sobre los medios se han desarrollado fuera de la academia? Algunas de estas tradiciones están, por supuesto, integradas en el sector comercial y otras tienen su origen en la labor de destacadas organizaciones religiosas, como la Iglesia católica. Historia de los Estudios sobre Medios fomenta la investigación que acerca estas historias a una conversación más cercana con las historias de los estudios académicos, pero alentamos sobre todo el trabajo de excavación de las tradiciones alternas de los grupos indígenas y de otros grupos subalternos y las prácticas culturales reflexivas que representan variedades menos reconocidas de estudios sobre medios - un cuerpo de trabajo en que han estado a la vanguardia investigadores latinoamericanos y otros estudiosos del Sur Global, a menudo en conexión con otros tipos de esfuerzos decoloniales. ${ }^{8}$

\section{Más allá del acceso abierto}

Los compromisos en la misión de la revista se extienden a su modo y forma de publicación. Historia de los Estudios sobre Medios es de acceso abierto (OA), lo que significa que los lectores no pagan por los artículos ni por las suscripciones. En este sentido, Historia de los Estudios sobre Medios se parece a muchas revistas nuevas, incluyendo a algunas lanzadas por las cinco grandes editoriales comerciales. La diferencia, crucial para nosotros, son las cuotas de autores: no cobramos ninguna, por principio. Muchas revistas de acceso abierto,
${ }^{6}$ Jean Comaroff y John Comaroff, Theory from the South: Or How Euro-America is Evolving toward Africa (Nueva York: Routledge, 2012), y Hanan Badr y Sarah Anne Ganter, "Towards Cosmopolitan Media and Communication Studies: Bringing Diverse Epistemic Perspectives into the Field", Global Media Journal (edición alemana) 11, no. 1 (2021).

${ }^{7}$ Sarah Anne Ganter y Félix Ortega, "The Invisibility of Latin American Scholarship in European Media and Communication Studies: Challenges and Opportunities of De-Westernization and Academic Cosmopolitanism", International Journal of Communication 13 (2019).

\footnotetext{
${ }^{8}$ Véase, por ejemplo, Claudia Magallanes Blanco y José Manuel Ramos Rodríguez, eds., Miradas propias: pueblos indígenas, comunicación y medios en la sociedad global (Quito: Ediciones CIESPAL, 2016), y Erick R. Torrico Villanueva, Hacia la comunicación decolonial (Sucre: Universidad Andina Simón Bolívar, 2016).
} 
incluso las publicadas por sociedades académicas, exigen a los autores el pago de una "cuota de tramitación del artículo" (APC) que suele estar entre 3 mil y 5 mil dólares. Creemos que el acceso abierto para los lectores no debe cambiarse por nuevas barreras a la autoría. En lugar de las cuotas de autores, financiamos nuestras operaciones a través de lo que comúnmente se denomina "financiación colectiva" (collective funding): el apoyo directo de bibliotecas y fundaciones. ${ }^{9}$ Lo que significa la financiación colectiva es que los envíos que recibimos se evalúan por sus méritos editoriales, sin tener en cuenta la riqueza personal o institucional. Entre otras cosas, este estatus de gratuidad apoya nuestra misión de publicar autores y temas de todo el mundo, ya que la mayoría de los académicos, más allá de un puñado de instituciones norteamericanas y de países europeos ricos, no puede pagar las APC.

En consonancia con nuestra política de gratuidad, interpretamos el "acceso abierto" de una manera más exigente y cargada de valores que el típico medio académico. Creemos que la propiedad y la gobernanza son importantes, que la publicación sostenible del acceso abierto debe hacerse sin ánimo de lucro, dirigida por la comunidad y de manera transparente. Junto con nuestra editorial dirigida por académicos, mediastudies.press, suscribimos los "Principios del auténtico acceso abierto" de Jean-Sebastian Caux. ${ }^{10}$ Nos comprometemos a utilizar una infraestructura abierta siempre que sea posible, incluyendo los programas mismos de publicación: PubPub, la plataforma de código abierto vinculada al MIT, con la premisa de recuperar la comunicación académica para la comunidad académica. ${ }^{11}$ Nuestra gobernanza y finanzas son transparentes y están abiertas al escrutinio, con acento especial en nuestras operaciones dirigidas por académicos. $^{12}$

Nuestro plan de limitar el volumen de artículos que publicamos —normalmente no más de diez al año- es un medio deliberado para alcanzar un fin orientado al valor. Como editores, podemos permitirnos adoptar un enfoque lento y cuidadoso con los autores y sus envíos. Consideramos que se trata de un ideal artesanal, opuesto conscientemente a la apurada ausencia de rostro que caracteriza a la mayoría de las revistas comerciales. ${ }^{13}$ Historia de los Estudios sobre Medios sustituye la edición artesanal y la revisión humana por pares por ScholarOne y la marea métrica. Los informes a nuestros autores, por ejemplo, no se limitan a recuentos de descargas y de citas sino también incluyen pasajes citados y contextos de citación.

La cuestión de la métrica plantea lo que es, para nosotros, un punto importante. Creemos que nuestra ética basada en el cuidado es compatible tanto con la calidad editorial como con las mejores prácticas en la publicación académica. Todos los artículos reciben
${ }^{9}$ Historia de los Estudios sobre Medios está participando en un nuevo modelo que integra a editores sin fines de lucro y bibliotecarios patrocinadores en una plataforma web compatible. La idea es que los bibliotecarios y otros financiadores puedan apoyar a los editores sobre la base de valores compartidos. La revista se encuentra entre los participantes del Programa de Inversión Comunitaria de Acceso Abierto (Open Access Community Investment Program, OACIP), organizado por el consorcio bibliotecario norteamericano LYRASIS. Acerca de la idea sobre los intercambios de financiamiento alineados a la misión, véase Jefferson Pooley, “Collective Funding to Reclaim Scholarly Publishing", The Commonplace, 16 de Agosto de 2021, https: //commonplace. knowledgefutures.org/pub/erpw9udj.

${ }^{10}$ Véase "Open Access Principles", mediastudies.press, https://www. mediastudies.press/oa-principles, y Jean-Sébastien Caux, "Genuine Open Access Principles", Jean-Sébastien Caux, https://jscaux.org/blog/post/ 2018/05/05/genuine- open-access/.

${ }^{11}$ PubPub es un proyecto del Knowledge Futures Group, sin fines de lucro, que surgió de la asociación entre la MIT Press y el MIT Media Lab. Véase "Our Mission", PubPub, https://www. pubpub.org/about. Sobre la visión de las publicaciones académicas del Knowledge Futures Group, véase Gabriel Stein et al., "Clarivate, ProQuest, and our Resistance to Commercializing Knowledge", The Commonplace, 18 de mayo de 2021, https: //commonplace. knowledgefutures.org/pub/kp8lylos/. ${ }^{12}$ Véase "Transparency", mediastudies.press, https://www. mediastudies. press/transparency.

${ }^{13}$ Para una aguda reflexión sobre la interacción entre la captura comercial y el propio malestar y capitulación de los editores tras la derrota, véase Mark Gibson, "Editing After ExitAlienation and Counter-Alienation in the Cultures of Cultural Studies Journals", Continuum 35, no. 3 (2021). 
una licencia Creative Commons y un DOI, y se pueden descargar en PDF y en otros siete formatos, incluido el XML JATS de lectura mecánica, con una indexación rápida y precisa en Google Scholar. ${ }^{14}$ Los correctores de la revista son también hábiles editores en línea, con créditos de cabecera y de artículo en reconocimiento a su vital labor.

Uno de nuestros objetivos es ayudar a ampliar el aspecto de un artículo académico. Elegimos la plataforma PubPub, en parte, por su amplio soporte de los formatos multimedia, con la idea de que los historiadores de los medios podrían iluminar el pasado de estos campos en diálogo con las nuevas formas de comunicación académica. Consideremos el típico artículo histórico basado en archivos: prevemos la publicación de documentos de archivo y otros soportes dentro de los artículos que los citan. También planeamos publicar colecciones de archivos y obras de dominio público medio olvidadas, con introducciones nuevas. Incluso volveremos a publicar trabajos arbitrados con licencia abierta aparecidos en otros lugares, con "Respuestas" enlazadas solicitadas para estos trabajos "superpuestos", así como para los artículos originales. La revista está abierta a la presentación de trabajos completos cuyos argumentos se presenten en audio, video y otras formas no textuales.

Los valores de cuidado lento de Historia de los Estudios sobre Medios también guían nuestro enfoque de la revisión por pares. Estamos comprometidos con un proceso de revisión humano y de desarrollo, con el objetivo de mejorar los manuscritos a través del intercambio colegiado. Inspirados por el ejemplo de Public Philosophy, vemos nuestro papel como algo más que revisores al servicio de una decisión puntual. ${ }^{15}$ Nuestro objetivo es cultivar en los revisores, también, un ethos de participación continua, de apoyo al autor y su manuscrito. Historia de los Estudios sobre Medios emplea por default la revisión doblemente anónima, pero fomenta modos más abiertos a discreción de los autores. Por ejemplo, apoyamos la revisión firmada, en la que los revisores firman sus comentarios y pueden seguir consultando con los autores a lo largo del proceso de revisión. También experimentaremos con la revisión comunitaria, en la que un borrador de un artículo se publica en una fase temprana del proceso, invitando a aportar comentarios firmados $-\mathrm{y}$ con revisiones iterativas respaldadas por el robusto soporte de versiones de PubPub. ${ }^{16}$

Entre nuestros objetivos está ayudar a fomentar una comunidad a distancia de estudiosos que trabajan en la historia de los estudios sobre medios y sus campos afines. Mantenemos, en cooperación con la revista, un grupo de trabajo sobre la historia de los estudios sobre medios. En cada sesión mensual, un investigador presenta un trabajo en proceso en una sesión remota en la que participan académicos de
${ }^{14}$ En efecto, Historia de los Estudios sobre Medios, durante su lanzamiento, atiende todos los criterios de cumplimiento del "Plan S" de los financiadores europeos, incluidos los requerimientos técnicos. Véase cOAlition S, "Plan S Principles", Plan S, https: //www. coalition-s.org/ plan_s_principles/. El editor de la revista, mediastudies.press, es miembro de Crossref, la Asociación de Publicaciones Académicas de Acceso Abierto (Open Access Scholarly Publishing Association, OASPA) y el colectivo Radical Open Access, con asociaciones verificadas que incluyen al Directory of Open Access Books (DOAB), el Proyecto MUSE y la OAPEN. Historia de los Estudios sobre Medios solicitará su incorporación en el listado del Directory of Open Access Journals (DOAJ) y su afiliación al Committee on Publication Ethics (COPE), cuando sea eligible después de un año de operación. Véase "About this Journal", History of Media Studies, 2021, https: $/ /$ hms.mediastudies. press/about.

${ }^{15}$ Véase "Formative Peer Review (FPR)", Public Philosophy Journal, https: //publicphilosophyjournal.org/ overview/.

\footnotetext{
${ }^{16}$ Véase "Peer Review", History of Media Studies, https://hms.mediastudies. press/peer-review.
} 
todo el mundo. La misma cultura de bienvenida y desarrollo informa al grupo de trabajo, como una extensión del espíritu editorial de la revista. ${ }^{17}$

\section{Una revista nueva}

Los quince ensayos breves de esta serie de lanzamiento, escritos por miembros del Consejo Editorial, reflejan los valores que hemos esbozado aquí. El compromiso de la revista de prestar atención a las inequidades y exclusiones estructurales se refleja en muchos de los ensayos de lanzamiento. Wendy Willems, en su contribución, señala una dolorosa ironía en los recientes intentos de des-occidentalizar y decolonizar los estudios sobre los medios, que a menudo han silenciado las historias reales del campo fuera del Norte Global y las luchas decoloniales de larga data de la diáspora africana. Ella advierte los riesgos de que la decolonización "se convierta en una metáfora vacía" y desafía a los historiadores de este campo a ir más allá de la mera inclusión para considerar "cómo el acto de incluir diferentes puntos de vista desafía, subvierte y problematiza" las concepciones dominantes del campo. Armond Towns cubre un terreno complementario, reviviendo una vigorosa tradición de los estudios negros que ha sido ocluida por los estudios que "situaban la vida de los negros únicamente como reacción al racismo blanco", y que puede renovarse mediante un "proyecto epistemológico alternativo que ... requeriría la reorganización del mundo tal y como se encuentra actualmente". Nos pide que leamos la historia de los estudios sobre medios y comunicación en paralelo con la historia de los estudios sobre negros y que veamos cómo algunas corrientes de los primeros "se desarrollaron con temor a la revolución negra y decolonial". Descentrando el Norte blanco y euroamericano desde perspectivas geo-intelectuales diferentes, Liu Hailong y Qin Yidan se preguntan: "¿Qué hubiera sido diferente si el estudio de la comunicación hubiera nacido en China?". Esto da paso a su debate sobre las particularidades del campo chino y las posibilidades de que su reciente giro hacia los medios incrustados en la experiencia china pueda marcar un nuevo comienzo.

Otros ensayos de la serie abordan el descentramiento geopolítico del Norte Global desde diferentes perspectivas. La contribución de Mohammad Ayish sitúa la historia de los estudios sobre medios en los contextos tecnológico, político y sociocultural del mundo árabe, siguiendo el cambio de un marco de desarrollo a uno de empoderamiento. El autor destaca los vínculos transnacionales y transregionales que desplazan al Estado-nación como locus histórico. En su cuidadoso examen de los estudios sobre medios en Argentina,
${ }^{17}$ El grupo de trabajo está alojado en el Consortium for History of Science, Technology and Medicine (CHSTM). Véase "Working Group on the History of Media Studies", History of Media Studies, https://hms . mediastudies. press/working-group. 
Mariano Zarowsky nos recuerda que "hablar de un campo de conocimiento" como el de los estudios sobre medios "implica estudiar un proceso de formación más que partir de entidades preexistentes". Lleva este proceso de formación al nivel del terreno, destacando la importancia de "las formaciones temporales y los contextos biográficos específi$\cos ^{\prime \prime}$, así como la interacción de las articulaciones regionales con los procesos globales. En su ensayo, Shiv Ganesh esboza un programa para un "enfoque centrado en el área" para la historia de los estudios sobre medios, con el propósito de desafiar los males gemelos de la "universalización teórica y el parroquialismo metodológico" en estos campos. Ilustra el valor del acercamiento con el caso del Sudeste Asiático, replanteando el terreno para encontrar alternativas a proyectar "cualquier cosa fuera de la historia euroamericana que se define en gran medida en términos de su diferencia". La biografía, el pensamiento y el rico legado de Jesús Martín-Barbero son el objeto del ensayo de Raúl Fuentes-Navarro. De los medios a las mediaciones de Martín-Barbero, argumenta Fuentes-Navarro, definiría a América Latina "como distinta entre las regiones culturales y lingüísticas occidentales". Una obra fundacional en el mundo iberoamericano que ha marcado el pensamiento desde su publicación en 1987, el libro también proporciona un mapa para la necesaria historiografía del campo en la región.

Muchos de los ensayos de lanzamiento se refieren a prescripciones y ajustes en el enfoque o la técnica historiográfica. Stefanie AverbeckLietz, por ejemplo, analiza un proyecto de periodismo sobre la Liga de las Naciones, para señalar el lugar marginal que ocupan los métodos históricos en los estudios de comunicación y medios en Alemania. En el camino, nos invita a reflexionar sobre los métodos con los que se escriben las historias del campo y las propias historias de esos métodos. Thomas Wiedemann y Michael Meyen, en su ensayo, reflexionan sobre un importante proyecto en idioma alemán -Biografisches Lexikon der Kommunikationswissenschaft o BLexKom - que ayudan a dirigir. En este sentido, se refieren a la promesa y al peligro historiográficos que la iniciativa ha puesto de manifiesto, incluyendo las cuestiones de quién cuenta la historia de quién. Sarah Cordonnier aborda temas similares en su estudio de la historiografía de la investigación francesa sobre los medios, identificando elisiones y "agujeros negros" en la literatura. Plantea grandes interrogantes sobre cómo escribir historias de un campo con tantas permutaciones, y si podemos encontrar la manera de ser "contemporáneos en la disciplina a pesar de todas las diferencias". En su contribución, Wendy Leeds-Hurwitz defiende los "grupos de teoría" como un prisma para hacer historia, que revela las infraestructuras sociales que apoyan la visibilidad y la influencia. Responde implícitamente a la cuestión del método que 
plantea Averbeck-Lietz, dejando espacio para el uso de la teoría de la comunicación de grupos para comprender la vida social de las ideas.

Otros ensayos señalan los deslices productivos de la etiqueta "estudios sobre medios" que la revista espera ampliar. Sue Collins, en su contribución, muestra cómo el estudio de la autoridad mediada expone los límites de lo que los "estudios de comunicación" o "estudios cinematográficos" por sí solos podrían hacer por nosotros. Aboga por que "la historia de la comunicación y los estudios sobre medios integren mejor a las historias del cine y de la cultura en su corpus". Con un espíritu similar, Filipa Subtil demuestra enérgicamente que la filosofía de la tecnología subyace de forma tácita a la historia de los estudios sobre medios, de manera que debería desafiar nuestras ideas preconcebidas sobre lo que son los medios. Nos insta a remediar una situación en la que, con algunas excepciones, "los historiadores de los estudios sobre medios no han prestado suficiente atención a la cuestión de la tecnología". Por su parte, Maria Löblich, de forma provocativa desdibuja las fronteras entre la historia de los estudios de comunicación y el fenómeno de la identidad colectiva, permitiendo la fertilización cruzada de estos dos campos académicos. El proyecto de Löblich, a la vez analítico y reconstructivo, nos permite pensar en "cómo los estudios de comunicación fueron atados históricamente a los sistemas simbólicos de la sociedad y qué grado de autonomía tenían". Por último, Ira Wagman nos desafía a "problematizar la historia de los 'estudios sobre medios' en tantos contextos como sea posible", tomando la Miranda Prorsus (encíclica papal de 1957 sobre el cine, la televisión y la radio), como una ilustración de la religión de uno de esos contextos. Al hacerlo, ofrece un excelente ejemplo de historias que examinan la conceptualización y el estudio de los medios fuera de los contextos académicos.

Tanto en lo individual como en conjunto, los ensayos de lanzamiento del Consejo Editorial ejemplifican dimensiones de la misión de la revista y la desarrollan de maneras que superan la imaginación de los editores que la formularon. La pluralidad tiene un potencial único. Aquí se manifiesta mediante escrituras que emergen de vidas académicas animadas por diversas problemáticas, estilos de pensamiento, lenguas, culturas políticas y contextos institucionales. Agradecemos sus creativas respuestas a nuestra invitación, que esperamos ustedes lean y hagan circular libremente, en el espíritu del acceso abierto.

\section{Bibliography}

"About this Journal". History of Media Studies. https://hms . mediastudies. press/about. 
Badr, Hanan y Sarah Anne Ganter. "Towards Cosmopolitan Media and Communication Studies: Bringing Diverse Epistemic Perspectives into the Field". Global Media Journal (edición alemana) 11, no. 1 (2021): 1-3. https://doi . org/10.22032/dbt . 49164.

Caux, Jean-Sébastien. “Genuine Open Access Principles". JeanSébastien Caux. https://jscaux.org/blog/post/2018/05/05/genuineopen-access.

cOAlition S. "Plan S Principles". Plan S. https : //www. coalition-s. org/plan_s_principles/

Comaroff, Jean y John Comaroff. Theory from the South: Or How EuroAmerica is Evolving toward Africa. Nueva York: Routledge, 2012.

Dutta, Mohan J. "Whiteness, Internationalization, and Erasure: Decolonizing Futures from the Global South". Communication and Critical/Cultural Studies 17, no. 2 (2020): 228-35. https: //doi.org/10.1080/14791420.2020.1770825.

Escobar, Arturo. Designs for the Pluriverse: Radical Interdependence, Autonomy, and the Making of Worlds. Durham, NC: Duke University Press, 2018.

"Formative Peer Review (FPR)". Public Philosophy Journal. https: //publicphilosophyjournal.org/overview/.

Ganter, Sarah Anne y Félix Ortega. "The Invisibility of Latin American Scholarship in European Media and Communication Studies: Challenges and Opportunities of De-Westernization and Academic Cosmopolitanism", International Journal of Communication 13 (2019): 68-91. https://ijoc.org/index.php/ijoc/article/ view/8449.

Gibson, Mark. "Editing After Exit-Alienation and Counter-Alienation in the Cultures of Cultural Studies Journals". Continuum 35, no. 3 (2021): 356-68. https://doi.org/10.1080/10304312.2021. 1902159.

Guillory, John. "Enlightening Mediation". En This is Enlightenment, editado por Clifford Siskin y William Warner, 37-66. Chicago: University of Chicago Press, 2010.

Magallanes Blanco, Claudia y José Manuel Ramos Rodríguez, eds. Miradas propias: pueblos indígenas, comunicación y medios en la sociedad global. Quito: Ediciones CIESPAL, 2016.

Martínez Guillem, Susana. "Sacando la Lengua in Rhetorical Theory and Criticism". Rhetoric, Politics \& Culture 1, no. I (2021): 45-54. https://muse.jhu.edu/article/801950.

“Open Access Principles”. mediastudies.press. https://www. mediastudies. press/oa-principles.

"Our Mission". PubPub. https://www. pubpub.org/about.

"Peer Review". History of Media Studies. https://hms. mediastudies. press/peer - review. 
Pooley, Jefferson. "Collective Funding to Reclaim Scholarly Publishing", The Commonplace. 16 de agosto de 2021. https:// commonplace. knowledgefutures.org/pub/erpw9udj.

Shechtman, Anna. "Command of Media's Metaphors". Critical Inquiry 47, no. 4 (2021): 644-74. https://doi.org/10. 1086/714512.

Stein, Gabriel, Travis Rich, Zach Verdin y Catherine Ahearn. "Clarivate, ProQuest, and our Resistance to Commercializing Knowledge", The Commonplace. 18 de mayo de 2021. https://commonplace. knowledgefutures.org/pub/kp81ylos/.

Suzina, Ana Cristina. "English as Lingua Franca: On the Sterilisation of Scientific Work", Media, Culture E Society 43, no. 1 (2021): 17179. https://doi.org/10.1177/0163443720957906.

Torrico Villanueva, Erick R. Hacia la comunicación decolonial. Sucre: Universidad Andina Simón Bolívar, 2016.

"Transparency". mediastudies.press. https://Www. mediastudies. press/transparency

Waisbord, Silvio. "Communication Studies without Frontiers? Translation and Cosmopolitanism across Academic Cultures". International Journal of Communication 10 (2016): 868-86. https: //ijoc.org/index.php/ijoc/article/view/3483.

"Working Group on the History of Media Studies". History of Media Studies. https://hms . mediastudies . press/working-group. 